\title{
Elevated Levels of Serum Neurofilament Light Chain Associated with Cognitive Impairment in Vascular Dementia
}

\author{
Weibin Ma, Jingjing Zhang, Jialei Xu $(\mathbb{D}$, Depeng Feng, Xiaoling Wang, and Fengyu Zhang \\ Department of Neurology, Liaocheng People's Hospital, Liaocheng, Shandong, China 252000 \\ Correspondence should be addressed to Jialei Xu; jialei621@163.com
}

Received 10 October 2020; Revised 20 October 2020; Accepted 22 October 2020; Published 2 November 2020

Academic Editor: Wen-Jun Tu

Copyright ( 2020 Weibin Ma et al. This is an open access article distributed under the Creative Commons Attribution License, which permits unrestricted use, distribution, and reproduction in any medium, provided the original work is properly cited.

\begin{abstract}
Objective. Vascular dementia $(\mathrm{VaD})$ is a progressive neurodegenerative disease with cognitive decline caused by cerebrovascular factors. Despite the great progress made in the past decade, VaD still lacks effective treatments and peripheral blood biomarkers. In this study, we tested the level of peripheral blood neurofilament light chain (NfL) in VaD patients and explored its relationship with cognitive impairment. Method. A total of 176 study subjects including 80 normal controls (NC) and 96 VaD patients were included in our study. Upon admission, we collected clinical and biochemical characteristics of all research subjects. We also evaluate the Montreal cognitive assessment scale (MoCA) scores of all subjects. The serum NfL level was measured by the single-molecule array (Simoa) method. Results. The years of education in the NC group and VaD group were $(11.65 \pm 3.04)$ years and $(10.53 \pm 3.87)$ years, respectively. Compared with VaD patients, the NC group has a higher level of education $(p=0.037)$. Furthermore, the results of Simoa indicated that VaD subjects had higher serum NfL levels compared with the NC group [ $(8.49 \pm 2.37) \mathrm{pg} / \mathrm{ml}$ vs. $(19.26 \pm 4.71) \mathrm{pg} / \mathrm{ml}, p<0.001]$. In terms of other clinical and biochemical characteristics, there was no significant difference between VaD and NC. The Spearman correlation analysis indicated that educational years have a significant positive correlation with MoCA scores $(r=0.238, p=0.041)$, while age and serum NfL levels have a significantly negative correlation with MoCA scores (age: $r=-0.213, p=0.040$; NfL: $r=-0.395, p=0.027$ ). However, further multiple regression analysis showed that only serum NfL level might serve as an independent risk factor for cognitive decline in $\operatorname{VaD}(\beta=0.317, p=0.021)$. Conclusion. The serum NfL levels in VaD subjects are significantly elevated, which may be used as a potential peripheral blood marker for predicting cognitive impairment in patients with $\mathrm{VaD}$.
\end{abstract}

\section{Introduction}

Vascular dementia $(\mathrm{VaD})$ can be defined as a neurodegenerative disease related to vascular brain injury, which is mainly manifested as cognitive decline and memory loss $[1,2]$. With the advent of a graying society, the number of people suffering from dementia has increased substantially. According to reports, the number of people diagnosed with dementia will double every 20 years [3]. Therefore, the number of dementia patients will reach 66 million in 2030 and 120 million in 2050 $[4,5]$. VaD accounts for about $15 \%-20 \%$ of all dementias, and its incidence is second only to Alzheimer's disease (AD) [6]. Unlike $\mathrm{AD}$, there is no licensed treatment for vascular dementia. In addition, $\mathrm{VaD}$ also lacks effective therapeutic targets $[7,8]$. Therefore, it is becoming imminent to find reliable molecular biomarkers to assess the potential risk of $\mathrm{VaD}$.
Neurofilaments (Nf) is a cytoskeletal protein mainly expressed in neurons, which belongs to Type IV intermediate filament family having structural similarity with some protein molecules such as nestin, peripherin, and a-internexin [9]. According to their molecular weight, $\mathrm{Nf}$ is biologically divided into three subunits: $68 \mathrm{kDa}$ Nf light (NfL), $160 \mathrm{kDa}$ $\mathrm{Nf}$ medium (NfM), and $205 \mathrm{kDa} \mathrm{Nf}$ heavy $(\mathrm{NfH})$ chains [10]. Recently, Nf is considered as a biomarker of neuroaxonal damage. When brain parenchyma is damaged, $\mathrm{Nf}$ is released into cerebrospinal fluid (CSF) and peripheral blood circulation $[11,12]$. The traditional enzyme-linked immunosorbent assay can only detect Nf in CSF, but the more accurate single-molecule array technology makes it possible to detect Nf in peripheral blood $[13,14]$.

Among the subunits of Nf, NfL is among the most promising biomarker candidates [15]. There is mounting evidence 
that NfL is related to a series of neurodegenerative diseases [16]. However, the relationship between NfL and VaD is still $\mathrm{dim}$. The purpose of this research is to detect the serum level of NfL and to further clarify whether NfL can be used as a potential biomarker for the prevention and treatment in $\mathrm{VaD}$.

\section{Patients and Methods}

2.1. Patient Population. The current study is a prospective cross-sectional descriptive design. Totally, 96 patients with $\mathrm{VaD}$ and 80 normal controls who were admitted to Liaocheng People's Hospital from June 2018 to May 2020 were recruited. Diagnosis of $\mathrm{VaD}$ was confirmed by attending neurologist according to the National Institute for Neurological Disorders and Stroke (NINDS-AIREN) and Diagnostic and Statistical Manual of Mental Disorders (DSM-V) [17, 18]. Exclusion criteria were as follows: (1) suffering from other types of dementia; (2) suffering from mental illness; (3) suffering from acute cerebrovascular disease or infectious disease; (4) malignant tumor; (5) history of surgery or other severe trauma within 3 months; (6) heart, liver, or kidney insufficiency and other serious acute and chronic diseases. This research abided by the Declaration of Helsinki. We obtained written consent from all subjects and approved by the Ethics Committee of Liaocheng People's Hospital.

2.2. Clinical and Biochemical Characteristics. At admission, clinical and biochemical characteristics were collected: education years, smoking and alcohol habits, coronary heart disease (CHD), high blood pressure (HBP), systolic blood pressure (SBP), diastolic blood pressure (DBP), hyperlipidemia (HLP), total cholesterol (TC), triglycerides (TG), highdensity lipoprotein cholesterol (HDL-C), low-density lipoprotein cholesterol (LDL-C), Diabetes Mellitus (DM), and fasting plasma glucose (FBG).

2.3. Cognitive Function Testing. Montreal cognitive assessment (MoCA) tool is a popular cognitive assessment tool having high sensitivity and specificity [19]. MoCA evaluation indicators include the following: alternating trail making, visuoconstructional skills, naming, memory, visuoconstructional skills attention, sentence repetition, verbal fluency, abstraction, delayed recall, and orientation. The MoCA evaluation takes about 10 minutes, and the total score is 30 points. One point is added to subjects who have received formal education for less than 12 years. A final total score of 26 and above is considered normal. The MoCA cognitive assessment is conducted under standard conditions by a professionally trained neurologist who is blind to the subjects' clinical baseline data.

2.4. Measurement of Serum NfL Levels. All subjects collected fasting serum samples within 24 hours of admission and stored them at $-80^{\circ} \mathrm{C}$. The serum levels of NfL were tested by the single-molecule array method (Simoa). The blood sample was placed at room temperature for 15 minutes and then centrifuged at $4^{\circ} \mathrm{C}$ for 15 minutes at a speed of $2000 \times$ $\mathrm{g}$ to obtain serum. The Simoa NF-light assay used commercial reagents (UmanDiagnostics, Umea, Sweden) on an
HD-1 platform (Neoline, Hangzhou, China) according to manufacturer's instructions. All samples were tested blindly, and the measurement was repeated [20].

2.5. Statistical Analysis. The concentration of NfL in serum showed a normal distribution. In this study, categorical variables are recorded by numbers (percentage, \%), while continuous variables are recorded by mean \pm standard deviation (mean $\pm \mathrm{SD}$ ). The $t$-test was applied for the comparison of continuous variables, and the chi-square test was applied for the comparison of categorical variables. Spearman's correlation analysis is used to assess the binary correlation. Multivariate regression analysis was applied to assess the predictive value of clinical and biochemical characteristics on the cognitive function in patients with $\mathrm{VaD}$. The SPSS 22.0 statistical software (SPSS Inc., IL, USA) was used in the study, and a $p$ value of 0.05 was considered significant.

\section{Results}

3.1. Clinical and Biochemical Characteristics. A total of 176 subjects including $96 \mathrm{VaD}$ patients and 80 normal controls admitted to Liaocheng People's Hospital from June 2018 to May 2020 were enrolled. The clinical and biochemical characteristics of all subjects were presented in Table 1. No significant differences were found in age, gender, smoking and alcohol habits, history of chronic disease (CHD, HBP, HLP, and DM), SBP, DBP, TC, TG, HDL-C, LDL-C, and FBG between $\mathrm{VaD}$ and $\mathrm{NC}$. However, significant differences were found in education years, MoCA scores, and serum NfL levels between the two groups (Figure 1).

3.2. Spearman's Correlation Analysis. The correlation between clinical and biochemical characteristics and MoCA score was assessed by Spearman's correlation analysis. The results of the correlation analysis are presented in Table 2. The results indicated that age $(r=-0.213, p=0.040)$ and the levels of serum NfL $(r=-0.395, p=0.027)$ were negatively correlated with MoCA scores in $\mathrm{VaD}$ patients, and the correlation was significant. The results also indicated that that the education years in $\mathrm{VaD}$ is positively correlated with the MoCA score $(r=0.238, p=0.041)$. However, in our current study, there is no significant correlation between cognitive decline and other clinical and biochemical characteristics of $\mathrm{VaD}$ patients $(p>0.05)$.

3.3. Multiple Regression Analysis. The results of multiple regression analysis of MoCA score and serum NfL level in $\mathrm{VaD}$ patients are presented in Table 3 . The results showed that serum NfL level may serve as an independent predictive risk factor for the cognitive decline in patients with $\mathrm{VaD}$. After adjusting for age, gender, years of education, SBP, DBP, TC, TG, HDL-C, LDL-C, FBG, and other clinical and biochemical characteristics, the serum NfL level still has important significance for the independent value of cognitive function in $\operatorname{VaD}(\beta=0.317, p=0.021)$. 
TABLE 1: Clinical and biochemical characteristics of all the subjects.

\begin{tabular}{|c|c|c|c|}
\hline Variables & $\mathrm{NC}$ & $\mathrm{VaD}$ & $p$ \\
\hline$N$ & 80 & 96 & - \\
\hline Age (years) & $69.59 \pm 5.31$ & $69.27 \pm 6.18$ & 0.742 \\
\hline Gender (male/female) & $49 / 31$ & $60 / 36$ & 0.865 \\
\hline Serum NfL (pg/mL) & $8.49 \pm 2.37$ & $19.26 \pm 4.71$ & $<0.001$ \\
\hline Education (years) & $11.65 \pm 3.04$ & $10.53 \pm 3.87$ & 0.037 \\
\hline Smoking $(n, \%)$ & $19(23.75)$ & $24(25.00)$ & 0.848 \\
\hline Alcohol $(n, \%)$ & $27(33.75)$ & $30(31.25)$ & 0.724 \\
\hline $\mathrm{CHD}(n, \%)$ & $10(12.50)$ & $13(13.54)$ & 0.838 \\
\hline $\operatorname{HBP}(n, \%)$ & $24(30.00)$ & $29(30.21)$ & 0.976 \\
\hline SBP (mmHg) & $134.46 \pm 14.28$ & $135.82 \pm 13.59$ & 0.519 \\
\hline DBP (mmHg) & $87.62 \pm 10.35$ & $88.17 \pm 11.63$ & 0.743 \\
\hline $\operatorname{HLP}(n, \%)$ & $17(21.25)$ & $20(20.83)$ & 0.946 \\
\hline $\mathrm{TC}(\mathrm{mmol} / \mathrm{L})$ & $4.72 \pm 0.93$ & $4.89 \pm 1.14$ & 0.286 \\
\hline $\mathrm{TG}(\mathrm{mmol} / \mathrm{L})$ & $1.64 \pm 0.31$ & $1.69 \pm 0.52$ & 0.451 \\
\hline HDL-C (mmol/L) & $1.26 \pm 0.39$ & $1.21 \pm 0.28$ & 0.325 \\
\hline LDL-C (mmol/L) & $2.41 \pm 0.67$ & $2.59 \pm 0.75$ & 0.098 \\
\hline $\mathrm{DM}(n, \%)$ & $14(17.50)$ & $16(16.67)$ & 0.884 \\
\hline FBG $(\mathrm{mmol} / \mathrm{L})$ & $6.59 \pm 1.16$ & $6.77 \pm 1.38$ & 0.356 \\
\hline MoCA (points) & $28.12 \pm 1.08$ & $21.57 \pm 3.40$ & $<0.001$ \\
\hline
\end{tabular}

NC: normal controls; VaD: vascular dementia; NfL: neurofilament light chain; CHD: coronary heart disease; HBP: high blood pressure; SBP: systolic blood pressure; DBP: diastolic blood pressure; HLP: hyperlipidemia; TC: total cholesterol; TG: triglycerides; HDL-C: highdensity lipoprotein cholesterol; LDL-C: low-density lipoprotein cholesterol; DM: Diabetes Mellitus; FBG: fasting plasma glucose; MoCA: Montreal cognitive assessment scale.

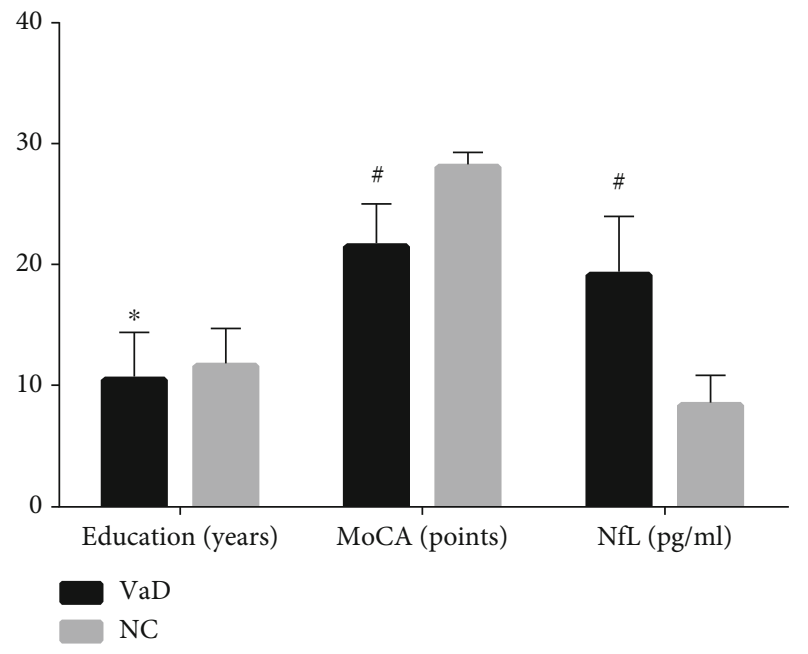

Figure 1: The differences in education years, MoCA scores, and serum NfL levels between $\mathrm{VaD}$ and NC. MoCA: Montreal cognitive assessment scale; NfL: neurofilament light chain; VaD: vascular dementia; NC: normal controls. Compared with NC group, ${ }^{*} p=0.037,{ }^{*} p<0.001$.
TABLE 2: Correlation coefficients between MoCA scores and various parameters in $\mathrm{VaD}$.

\begin{tabular}{lcc}
\hline & \multicolumn{2}{c}{ MoCA (points) } \\
\hline Age (years) & -0.213 & $p$ \\
Gender & 0.301 & 0.040 \\
Education (years) & 0.238 & 0.454 \\
SBP $(\mathrm{mmHg})$ & -0.372 & 0.041 \\
DBP $(\mathrm{mmHg})$ & -0.526 & 0.245 \\
TG $(\mathrm{mmol} / \mathrm{L})$ & -0.377 & 0.418 \\
TC $(\mathrm{mmol} / \mathrm{L})$ & -0.429 & 0.183 \\
HDL-C (mmol/L) & 0.284 & 0.204 \\
LDL-C (mmol/L) & -0.267 & 0.312 \\
FBG (mmol/L) & -0.482 & 0.059 \\
Serum NfL (pg/mL) & -0.395 & 0.656 \\
\hline
\end{tabular}

MoCA: Montreal cognitive assessment scale; VaD: vascular dementia; SBP: systolic blood pressure; DBP: diastolic blood pressure; TC: total cholesterol; TG: triglycerides; HDL-C: high-density lipoprotein cholesterol; LDL-C: low-density lipoprotein cholesterol; FBG: fasting plasma glucose; NfL: neurofilament light chain.

TABLE 3: Association between MoCA scores and various parameters in $\mathrm{VaD}$.

\begin{tabular}{lccc}
\hline & Regression coefficient & $p$ & $95 \%$ CI \\
\hline Age (years) & 0.138 & 0.184 & $0.725-1.203$ \\
Gender & 0.263 & 0.196 & $0.788-1.194$ \\
Education (years) & 0.214 & 0.086 & $0.672-1.145$ \\
SBP (mmHg) & 0.386 & 0.437 & $0.783-1.092$ \\
DBP (mmHg) & 0.379 & 0.322 & $0.561-1.236$ \\
TG (mmol/L) & 0.272 & 0.195 & $0.826-1.215$ \\
TC $(\mathrm{mmol} / \mathrm{L})$ & 0.305 & 0.618 & $0.487-1.309$ \\
HDL-C (mmol/L) & 0.403 & 0.239 & $0.799-1.167$ \\
LDL-C (mmol/L) & 0.260 & 0.193 & $0.915-1.128$ \\
FBG (mmol/L) & 0.621 & 0.264 & $0.871-1.053$ \\
Serum NfL (pg/mL) & 0.317 & 0.021 & $1.634-2.481$ \\
\hline
\end{tabular}

MoCA: Montreal cognitive assessment scale; VaD: vascular dementia; SBP: systolic blood pressure; DBP: diastolic blood pressure; TC: total cholesterol; TG: triglycerides; HDL-C: high-density lipoprotein cholesterol; LDL-C: low-density lipoprotein cholesterol; FBG: fasting plasma glucose; NfL: neurofilament light chain.

\section{Discussion}

The aim of the research was to study the relationship between cognitive function and serum NfL levels in $\mathrm{VaD}$ patients as well as normal controls. The results showed that the serum $\mathrm{NfL}$ level in $\mathrm{VaD}$ patients was significantly higher than that in the normal control group, while the MoCA score was lower. We also found that the MoCA score in $\mathrm{VaD}$ was significantly negatively correlated with serum NfL levels and age and positively correlated with years of education but had no significant correlation with other clinical and biochemical characteristics. This association is not affected by disturbing factors such as age, gender, years of education, 
SBP, DBP, TC, TG, HDL-C, LDL-C, and FBG. To our knowledge, we have confirmed for the first time that serum NfL may serve as an independent risk factor for cognitive impairment in patients with $\mathrm{VaD}$.

It has recently been discovered that NfL is related to a battery of neurological diseases. Johanna Gaiottino and his colleagues found that patients with $\mathrm{AD}$, amyotrophic lateral sclerosis (ALS), and Guillain-Barré-syndrome (GBS) have higher levels of cerebrospinal fluid and serum NfL, and this change in NfL level is not accompanied by evidence of structural damage of central nervous system (CNS) [21]. ChingHua Lu's team further confirmed that serum NfL level was an easily accessible marker for prognostic of patients with ALS [22]. Interestingly, NfL mutations can cause severe early onset of Charcot-Marie-Tooth (CMT) disease [23]. In addition, elevated levels of NfL in cerebrospinal fluid or peripheral blood have also been reported in Parkinson's disease, relapsing-remitting multiple sclerosis, progressive supranuclear palsy, and brain metastases [24-28]. All the above studies suggested that NfL can be used as a biological target of certain nervous system disorders.

Emerging evidence indicates that NfL is involved in the pathological mechanism of cognitive declines [29]. A Chinese Taipei study showed that plasma NfL is a biomarker of cognitive decline in $\mathrm{AD}$ and Parkinson's disease (PD), and it is more specific for AD [24]. A 5-year longitudinal retrospective study in the United States showed that higher serum NfL levels are related to poor current and future clinical and cognitive performance [30]. In hereditary frontotemporal dementia, blood NfL can be used as a biomarker of disease progression, and the longitudinal measurement of NfL provides valuable information as a marker of treatment effect [31]. Not only in frontotemporal dementia, a Swedish study showed that plasma NfL is a noninvasive biomarker for $\mathrm{AD}$ [32]. Although the relationship between NfL and cognitive impairment has been widely reported, its mechanism is not completely clear.

Our research has some limitations. Firstly, the current study is a single-center study with a small sample size. Secondly, we did not monitor the serum NfL level longitudinally nor did we dynamically follow up the cognitive function and prognosis of patients. Thirdly, because the gold standard for diagnosis of $\mathrm{VaD}$ is biopsy, thus, our diagnosis for $\mathrm{VaD}$ may not be accurate enough, and $\mathrm{VaD}$ patients may also accompany by other types of cognitive impairment. However, our study confirmed for the first time the correlation between elevated serum NfL levels and cognitive decline, which has important clinical potential application value in $\mathrm{VaD}$.

\section{Conclusion}

In conclusion, our study mainly found that the serum NfL levels of $\mathrm{VaD}$ patients were significantly elevated than that of the NC group. Our study reported for the first time that serum NfL plays an important role in the pathophysiology of the cognitive function of $\mathrm{VaD}$ patients. We expect the emergence of larger multicenter studies to confirm the association between serum NfL concentrations and cognitive function in patients with $\mathrm{VaD}$. Elucidating the potential pathological mechanism that NfL involved in the pathogenesis of $\mathrm{VaD}$ will have great applied value.

\section{Data Availability}

The data used to support the findings of this study are available from the corresponding author upon request.

\section{Conflicts of Interest}

The authors declare that they have no any conflict of interests.

\section{Authors' Contributions}

Weibin Ma and Jingjing Zhang are cofirst authors, and they contributed equally to this work.

\section{Acknowledgments}

All the authors are grateful to the Department of Neurology, Liaocheng People's Hospital.

\section{References}

[1] J. Li, S. Li, Y. Song et al., "Association of serum FAM19A5 with cognitive impairment in vascular dementia," Disease Markers, vol. 2020, Article ID 8895900, 5 pages, 2020.

[2] Q. Wang, W. Yang, J. Zhang, Y. Zhao, and Y. Xu, "TREM2 overexpression attenuates cognitive deficits in experimental models of vascular dementia," Neural Plasticity, vol. 2020, Article ID 8834275, 10 pages, 2020.

[3] Y. Xu, Q. Wang, Z. Qu, J. Yang, X. Zhang, and Y. Zhao, "Protective effect of hyperbaric oxygen therapy on cognitive function in patients with vascular dementia," Cell Transplantation, vol. 28, no. 8, pp. 1071-1075, 2019.

[4] Q. Wang, Y. Xu, C. Qi, A. Liu, and Y. Zhao, "Association study of serum soluble TREM2 with vascular dementia in Chinese Han population," The International Journal of Neuroscience, vol. 130, no. 7, pp. 708-712, 2020.

[5] Y. Xu, Q. Wang, R. Cui, K. Lu, Y. Liu, and Y. Zhao, "Uric acid is associated with vascular dementia in Chinese population," Brain and Behavior, vol. 7, no. 2, article e00617, 2017.

[6] J. O'Brien and A. Thomas, "Vascular dementia," The Lancet, vol. 386, no. 10004, pp. 1698-1706, 2015.

[7] K. Shao, S. Shan, W. Ru, and C. Ma, "Association between serum NPTX2 and cognitive function in patients with vascular dementia," Brain and Behavior, vol. 10, article e01779, 2020.

[8] Y. Xu, Q. Wang, Y. Liu, R. Cui, K. Lu, and Y. Zhao, "Association between Helicobacter pylori infection and carotid atherosclerosis in patients with vascular dementia," Journal of the Neurological Sciences, vol. 362, pp. 73-77, 2016.

[9] B. A. Gordon, "Neurofilaments in disease: what do we know?," Current Opinion in Neurobiology, vol. 61, pp. 105-115, 2020.

[10] R. Perrot, R. Berges, A. Bocquet, and J. Eyer, "Review of the multiple aspects of neurofilament functions, and their possible contribution to neurodegeneration," Molecular Neurobiology, vol. 38, no. 1, pp. 27-65, 2008.

[11] M. Duering, M. J. Konieczny, S. Tiedt et al., "Serum neurofilament light chain levels are related to small vessel disease burden," Journal of Stroke, vol. 20, no. 2, pp. 228-238, 2018. 
[12] S. Mariotto, E. Sechi, and S. Ferrari, "Serum neurofilament light chain studies in neurological disorders, hints for interpretation," Journal of the Neurological Sciences, vol. 416, p. 116986, 2020.

[13] M. Khalil, C. E. Teunissen, M. Otto et al., "Neurofilaments as biomarkers in neurological disorders," Nature Reviews Neurology, vol. 14, no. 10, pp. 577-589, 2018.

[14] C. Meregalli, G. Fumagalli, P. Alberti et al., "Neurofilament light chain: a specific serum biomarker of axonal damage severity in rat models of Chemotherapy-Induced Peripheral Neurotoxicity," Archives of Toxicology, vol. 94, no. 7, pp. 2517-2522, 2020.

[15] F. Taghdiri, N. Multani, M. Ozzoude et al., "Neurofilamentlight in former athletes: a potential biomarker of neurodegeneration and progression," European Journal of Neurology, vol. 27, no. 7, pp. 1170-1177, 2020.

[16] Y. Lee, B. H. Lee, W. Yip, P. Chou, and B.-S. Yip, "Neurofilament proteins as prognostic biomarkers in neurological disorders," Current Pharmaceutical Design, vol. 25, pp. 4560-4569, 2019.

[17] T. Erkinjuntti, "Clinical criteria for vascular dementia: the NINDS-AIREN criteria," Dementia and Geriatric Cognitive Disorders, vol. 5, pp. 189-192, 2004.

[18] F. Edition, Diagnostic and statistical manual of mental disorders, Am Psychiatric Assoc, 2013.

[19] Y. Xu, Q. Wang, Y. Liu, R. Cui, and Y. Zhao, "Is Helicobacter pylori infection a critical risk factor for vascular dementia?," The International Journal of Neuroscience, vol. 126, no. 10, pp. 899-903, 2016.

[20] Q.-F. Li, Y. Dong, L. Yang et al., "Neurofilament light chain is a promising serum biomarker in spinocerebellar ataxia type 3," Molecular Neurodegeneration, vol. 14, pp. 1-8, 2019.

[21] J. Gaiottino, N. Norgren, R. Dobson et al., "Increased neurofilament light chain blood levels in neurodegenerative neurological diseases," PLoS One, vol. 8, no. 9, article e75091, 2013.

[22] C.-H. Lu, C. Macdonald-Wallis, E. Gray et al., "Neurofilament light chain: a prognostic biomarker in amyotrophic lateral sclerosis," Neurology, vol. 84, no. 22, pp. 2247-2257, 2015.

[23] A. Jordanova, P. De Jonghe, C. Boerkoel et al., "Mutations in the neurofilament light chain gene (NEFL) cause early onset severe Charcot-Marie-Tooth disease," Brain : A Journal of Neurology, vol. 126, no. 3, pp. 590-597, 2003.

[24] Y.-S. Lin, W.-J. Lee, S.-J. Wang, and J.-L. Fuh, "Levels of plasma neurofilament light chain and cognitive function in patients with Alzheimer or Parkinson disease," Scientific Reports, vol. 8, pp. 1-8, 2018.

[25] J. Kuhle, G. Disanto, J. Lorscheider et al., "Fingolimod and CSF neurofilament light chain levels in relapsing-remitting multiple sclerosis," Neurology, vol. 84, no. 16, pp. 1639-1643, 2015.

[26] J. C. Rojas, A. Karydas, J. Bang et al., "Plasma neurofilament light chain predicts progression in progressive supranuclear palsy," Annals of Clinical and Translational Neurology, vol. 3, no. 3, pp. 216-225, 2016.

[27] N. D. Le, L. Muri, D. Grandgirard, J. Kuhle, D. Leppert, and S. L. Leib, "Evaluation of neurofilament light chain in the cerebrospinal fluid and blood as a biomarker for neuronal damage in experimental pneumococcal meningitis," Journal of Neuroinflammation, vol. 17, pp. 1-9, 2020.

[28] A. Winther-Larsen, C. V. B. Hviid, P. Meldgaard, B. S. Sorensen, and B. Sandfeld-Paulsen, "Neurofilament light chain as a biomarker for brain metastases," Cancers, vol. 12, no. 10, p. 2852, 2020.

[29] K. E. Osborn, O. A. Khan, H. A. Kresge et al., "Cerebrospinal fluid and plasma neurofilament light relate to abnormal cognition," Alzheimer's \& Dementia: Diagnosis, Assessment \& Disease Monitoring, vol. 11, no. 1, pp. 700-709, 2019.

[30] D. Jakimovski, R. Zivadinov, M. Ramanthan et al., "Serum neurofilament light chain level associations with clinical and cognitive performance in multiple sclerosis: a longitudinal retrospective 5-year study," Multiple Sclerosis Journal, vol. 26, no. 13 , pp. 1670-1681, 2020.

[31] L. H. Meeter, E. G. Dopper, L. C. Jiskoot et al., "Neurofilament light chain: a biomarker for genetic frontotemporal dementia," Annals of Clinical and Translational Neurology, vol. 3, no. 8, pp. 623-636, 2016.

[32] N. Mattsson, U. Andreasson, H. Zetterberg, and K. Blennow, "Association of plasma neurofilament light with neurodegeneration in patients with Alzheimer disease," JAMA Neurology, vol. 74, no. 5, pp. 557-566, 2017. 